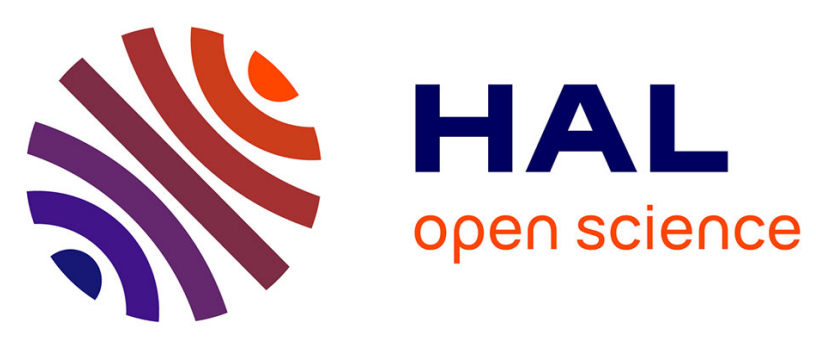

\title{
Comparison of viraemia-, clinical-based estimates of within- and between pen transmission of Classical Swine Fever Virus from three transmission experiments
}

Benoit Durand, Sylvie Davila, Roland Cariolet, Alain Mesplede, Marie-Frédérique Le Potier

\section{- To cite this version:}

Benoit Durand, Sylvie Davila, Roland Cariolet, Alain Mesplede, Marie-Frédérique Le Potier. Comparison of viraemia-, clinical-based estimates of within- and between pen transmission of Classical Swine Fever Virus from three transmission experiments. Veterinary Microbiology, 2009, 135 (3-4), pp.196. 10.1016/j.vetmic.2008.09.056 . hal-00532501

\section{HAL Id: hal-00532501 \\ https://hal.science/hal-00532501}

Submitted on 4 Nov 2010

HAL is a multi-disciplinary open access archive for the deposit and dissemination of scientific research documents, whether they are published or not. The documents may come from teaching and research institutions in France or abroad, or from public or private research centers.
L'archive ouverte pluridisciplinaire HAL, est destinée au dépôt et à la diffusion de documents scientifiques de niveau recherche, publiés ou non, émanant des établissements d'enseignement et de recherche français ou étrangers, des laboratoires publics ou privés. 


\section{Accepted Manuscript}

Title: Comparison of viraemia-, clinical-based estimates of within- and between pen transmission of Classical Swine Fever Virus from three transmission experiments

Authors: Benoit Durand, Sylvie Davila, Roland Cariolet, Alain Mesplede, Marie-Frédérique Le Potier

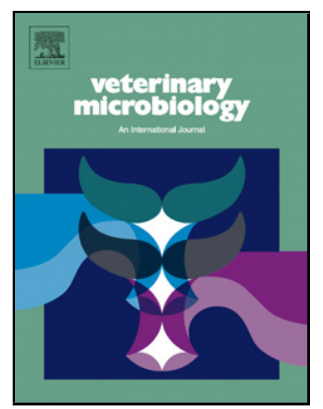

PII: S0378-1135(08)00430-6

DOI: doi:10.1016/j.vetmic.2008.09.056

Reference: VETMIC 4196

To appear in: $\quad$ VETMIC

Received date: 26-11-2007

Revised date: $\quad 20-8-2008$

Accepted date: $\quad$ 15-9-2008

Please cite this article as: Durand, B., Davila, S., Cariolet, R., Mesplede, A., Le Potier, M.-F., Comparison of viraemia-, clinical-based estimates of within- and between pen transmission of Classical Swine Fever Virus from three transmission experiments, Veterinary Microbiology (2008), doi:10.1016/j.vetmic.2008.09.056

This is a PDF file of an unedited manuscript that has been accepted for publication. As a service to our customers we are providing this early version of the manuscript. The manuscript will undergo copyediting, typesetting, and review of the resulting proof before it is published in its final form. Please note that during the production process errors may be discovered which could affect the content, and all legal disclaimers that apply to the journal pertain. 
1 Comparison of viraemia- and clinical-based estimates of within- and between pen transmission of Classical Swine Fever Virus from three transmission experiments.

4 Benoit DURAND ${ }^{1}$, Sylvie DAVILA ${ }^{2}$, Roland CARIOLET ${ }^{2}$, Alain MESPLEDE $^{2, * *}$., Marie5 Frédérique LE POTIER ${ }^{2, *}$

6

$7{ }^{1}$ AFSSA-LERPAZ, Unité Epidémiologie, 22 rue Pierre Curie, BP 67, F-94703 Maisons8 Alfort

$9 \quad{ }^{2}$ AFSSA-LERAPP, Unité Virologie Immunologie Porcines Ploufragan, Zoopole, BP 53, F1022440 Ploufragan

11

12 * Corresponding author, tel: +33 2960162 90, fax: +33 2960162 23, e-mail:

13 mf.lepotier@afssa.fr

$14^{* *}$ Current address : Laboratoire vétérinaire des Landes, LVD40, F-40000 Mont de Marsan 
Abstract.

Analyses of recent classical swine fever (CSF) epidemics in the European Union have shown that silent circulation of CSF virus (CSFV) occurs before the first outbreak is detected and this may lead to a large epidemic. However, severity of CSF disease signs may be linked with efficacy of disease transmission, the most severely affected animals having a higher infectivity than the less affected ones. The purpose of this study was to combine disease transmission quantification methods with CSF clinical signs quantification tools to investigate whether clinical signs, considered as infectivity markers, may allow us to calculate reliable estimates for disease transmission parameters. Data from three transmission experiments were used, varying according to the viral strain (Eystrup or Paderborn) and to the contact structure between experimentally inoculated and contact animals (direct or indirect contact). Withinand between-pen basic reproduction ratios (R0) were compared using viraemia data or clinical data. Between-pen R0 estimates were close and not significantly $>1$, with either strain or computation mode (using viraemia or clinical data). Conversely, within-pen R0s (Paderborn strain) computed using clinical data appeared higher than the estimates obtained using

31 viraemia data. A models comparison (Bayes information criterion) showed a better fit of the 32 clinical-based models, for both strains. This suggests that, in affected herds, the most severely affected animals could play a prominent role in CSFV transmission.

Keywords. classical swine fever, pig, basic reproduction ratio, clinical score 


\section{Introduction}

Classical swine fever (CSF) is a highly contagious viral disease of worldwide importance. Domestic and feral pigs are the only natural reservoir of Classical Swine Fever Virus (CSFV). The disease is endemic in many countries of Eastern Europe, South-Eastern Asia, and Southern and Central America (Dahle and Liess 1992, Moennig 2000). When introduced in a fully susceptible population, CSFV may cause large epidemics. In 1980 the European Union (EU) adopted an eradication strategy in pig herds, based upon a stamping-out policy. Initially this policy involved mass vaccination but, from 1992 onwards was implemented without preventive vaccination. However, emergency vaccination was still possible in large outbreaks. In many EU countries currently, CSF has been eradicated from the domestic pig population (Anon., 2007) but it remains endemic in some wild boar populations, posing a risk of outbreaks on farms (Fritzemaier et al. 2000, Fröhlich et al. 2002).

CSF outbreaks cause huge economic losses and have important consequences for the pig industry, especially in densely populated areas where the virus may spread quickly (Meuwissen et al. 1999, Saatkamp et al. 2000). Early detection of CSFV circulation is therefore crucial for minimizing epidemic size and economic consequences. Recent outbreaks have shown how a prolonged silent circulation of CSFV in pig herds may result in large epidemics (Elbers et al. 1999). Vague and non-specific disease symptoms may allow virus to spread unnoticed (Koenen et al. 1996, Sandvik et al. 2000, Terpstra and De Smit 2000). Severity of clinical signs is known to vary according to the CSFV strain, but also according to several other factors such as age, breed, health and immune status (Depner et al. 1997, Floegel-Niesmann et al. 2003, Moennig et al. 2003). Subacute or chronic disease courses observed in recent outbreaks pose real diagnostic problems (Elbers et al. 2004, Engel et al. 2005). However, the severity of clinical signs is probably linked with disease transmission. 
The most severely affected animals are also probably the most contagious animals, as suspected for other pig diseases such as foot and mouth disease (Alexandersen et al. 2003, Quan et al. 2004). There is also a correlation between virus growth in cell culture and severity of disease caused. In vitro results show the amount of virus released in cell culture media is higher when cells are infected with strains causing more severe clinical signs compared to those causing less severe disease (Mittelholzer et al. 2000). Thus less severe disease forms could be associated with a lower disease transmission and a slower CSFV spreading in cells.

Few studies have been conducted to quantify the severity of CSF clinical signs. A first clinical scoring system was developed by Wood et al. (1988) to quantify the virulence of a specific strain. More recently, Mittelholzer et al. (2000) proposed a general and practical scoring system based upon a semi-quantitative judgement of 10 CSF-relevant criteria using scores varying from 0 to 3 . They used this score to compare the clinical signs provoked by several CSFV isolates in SPF animals. Floegel-Niesman et al. (2003) added to these clinical scores a pathological score to quantify lesions, and used both scores to compare recent CSFV isolates, with isolates obtained during the 1990's.

The basic reproduction ratio (R0) is the most widely used parameter to quantify the transmission of an infection. It is defined as the average number of secondary infected individuals caused by a single typical infectious individual in an infinite susceptible population (Anderson and May 1991). If R0 is smaller than 1, an infectious animal infects, on average, less than one susceptible animal and the infection process wanes. Conversely if R0 is greater than 1, the infection spreads, and a large epidemic can occur. Methods designed to estimate R0 from experimental transmission data are either based on the sole final state of the animals (infected or not) (Kroese and De Jong 2001) or on a reconstruction of the withingroup contagion process. In this method for each animal, an infection date and a period during which the animal has been infectious are computed (Becker 1989, De Jong and Kimman 
1994, Klinkenberg et al. 2002). Several CSF R0 estimates based upon transmission experiments have been published (Dewulf et al. 2002, Laevens et al. 1998, Laevens et al. 1999, Dewulf et al. 2001). In these the within-group contagion process was reconstructed using results of CSFV isolation from blood: an animal was considered as infectious as long as it was viraemic, and individual infection dates were computed assuming a latent period (between infection and the beginning of viraemia) of fixed duration. These were either estimated from values observed in experimentally inoculated animals (Laevens et al. 1998 and 1999), or fixed as a parameter (Klinkenberg et al. 2002).

The objective of this study was to investigate whether CSFV clinical signs, considered as infectivity markers, may enable reliable estimates of disease transmission parameters to be estimated.

We compared the R0 estimates based on viraemia, with the values obtained by quantification of the clinical signs using the scoring system proposed by Mittelholzer et al. (2000). We used data from three transmission experiments conducted in 2001 using two strains with different virulence levels. Method- and strain-specific R0s estimates were calculated, and the differences are discussed.

\section{Materials and Methods}

\subsection{Cells and virus}

The highly-virulent Eystrup strain, genetic subtype 1.1 was kindly provided by A. Summerfield, Institute of Virology and Immunoprophylaxis, Mitterhäusern, Switzerland; with the permission of H.J. Thiel, Institute of Virology, University of Giessen, Germany. The moderately virulent Paderborn strain (CSF0277), genetic subtype 2.1, was kindly provided by the Community Reference laboratory (CRL) for CSF, Hanover, Germany). The strains were 
propagated twice on PK15 cells (obtained from the CRL, Hanover, Germany) as

110 recommended (Anon., 2002).

\subsection{Transmission experiments}

Experimental procedures and animal management were undertaken according to the

114 French legislation on animal experimentation (registration number 22-17).

Three transmission experiments were conducted in 2001 in the Level 3 protected facilities at AFSSA -Ploufragan, using two different CSFV strains. The Eystrup strain was used in an indirect transmission experiment (A) whereas the Paderborn strain was used both in an indirect (B) and a direct contact transmission experiment (C).

Eight week old specific pathogen free (SPF) and pestivirus-free Large-White pigs weighing $35 \mathrm{~kg}$ and originating from the AFSSA protected facilities of Ploufragan were used.

121 Experimentally inoculated animals received an oronasal dose of $10^{6} \mathrm{TCID}_{50}$ (tissue culture $50 \%$ infective dose) CSFV ( $2 \mathrm{ml}$ per nostril). Control pigs received $4 \mathrm{mls}$ of culture medium oronasally instead of virus.

Two different experimental designs were used, with two pens (numbered 1 and 2). In experiments A and B, 5 experimentally inoculated pigs were placed in pen 1, and 4 contact pigs in pen 2. Both pens were physically separated, and animal care was organized so that only airborne transmission between infected and non-infected animals could occur. In 128 experiment C, 2 experimentally inoculated pigs and 3 contact pigs were placed in each pen.

129 Separation between pens was removed to allow direct contacts between animals. The three 130 experiments thus differed according to the virus strain and to the studied transmission mode.

131 In experiment A: Eystrup strain was used to study between-pen transmission. In experiment

132 B: Paderborn strain was used to study between-pen transmission, and in experiment C:

133 Paderborn strain was used to study within-pen transmission. As the design for experiment C 
134 allowed direct contacts between the two pens, data from this experiment were treated as if all

135 of the 10 animals had shared the same pen.

136 Pigs were examined daily using the clinical scoring system proposed by Mittelholzer

137 et al. (2000). This consists of 10 criteria scored from 0 to 3 according to the severity of the

138 symptoms (0: no symptoms, 3: severe symptoms). Scores are added to obtain an individual

139 clinical score (CS). Of the 10 criteria proposed by Mittelholzer et al. (2000), the $10^{\text {th }}$

140 (leftovers in feeding troughs) could not be determined, since in our facilities the animals are

141 fed ad libitum in a common trough. The measured CSs thus ranged from 0 to 27 , instead of 0 142 to 30.

143 Blood samples were taken from the jugular vein in heparin sterile tubes before the

144 experimental inoculation (day 0 ), and on days $3,5,7,10,12,14,17,19,21,24,26,28$

145 (experiment A); 3, 5, 7, 10, 12, 14, 17, 19, 21, 24, 26, 28, 31, 33, 35, 38, 40, 42 (experiment

146 B); and 3, 7, 9, 11, 14, 16, 18, 12, 23, 25, 27, 28, 31, 35, 38, 45 (experiment C). Samples were

147 missing for 3 pigs on D24 (animals 6903, 6884, 6909) and for one animal on D33 (pig 6886).

148 Blood was inoculated into wells containing confluent PK15 cell monolayers. After two

149 passages, the supernatants were removed and the cells were fixed with cold acetone (80\%).

150 Diluted pig anti-CSFV antibodies followed by a mouse FITC conjugate were added to 151 identify virus presence by fluorescence microscopy (Anon. 2002).

Pigs were followed-up (clinical scores and CSFV isolation from blood) until the

153 disappearance of the clinical signs and the end of the viraemic period at day 28 (experiment

154 A), 42 (experiment B) and 45 (experiment C). All the pigs were euthanised by intraveinous

155 booster of sodium pentobarbital at the end of the experiment or earlier if needed for welfare 156 reasons. 


\subsection{Virological- and clinical-based datasets}

For each of the three experiments, three different methods were used to reconstruct the contagion process from raw data, resulting in three different datasets: a viraemia-based dataset and two clinical-based datasets.

The viraemia-based dataset was analogous to the datasets used in previous studies (Laevens et al. 1998 and 1999, Klinkenberg et al., 2002). Missing data (days without CSFV isolation assay) were first linearly interpolated from the nearest observations. Infection dates were then calculated from the date of the $1^{\text {st }} \mathrm{CSFV}$ isolation from blood, assuming a latent period (between infection and the $1^{\text {st }}$ CSFV isolation from blood) of fixed duration. This latent period was estimated from the average duration between infection and the $1^{\text {st }}$ CSFV isolation from blood observed in experimentally inoculated animals. Animal infectivity was also based upon results of CSFV isolation from blood, an animal being considered as infectious as long as it was viraemic.

The two clinical-based datasets were derived from the CSs. In both cases, infection dates were based upon the $1^{\text {st }}$ day with a $\mathrm{CS}>0$, using again a fixed-length latent period (between infection and the $1^{\text {st }}$ day with $\mathrm{CS}>0$ ). The duration of this latent period was estimated from the average value observed in experimentally inoculated animals. The two clinical-based datasets differed according to how individual infectivity was derived from the clinical signs. These treated CSs as a quantitative or as a qualitative variable. For the quantitative clinical-based dataset, animal infectivity was directly based on CSs: at a given time point, the infectivity of an animal was assumed to be measured by its CS. Conversely, for the qualitative clinical-based dataset, an animal was considered as infectious as long as its CS was $>0$.

Whatever the dataset, the duration of the latent period was assumed to be strainspecific and was thus calculated once for the Eystrup strain (using data from experimentally 
184 inoculated animals, experiment A) and once for the Paderborn strain (using data from

185 experimentally inoculated animals, experiments B and C).

186 For each experiment, pen and time step, the following variables were calculated: the

187 number of animals and the number of uninfected animals in the pen at the beginning of the

188 time step, the number of contact animals infected during the time step, and the infectivity

189 released by animals located in the pen, during the time step. For the viraemia-based dataset

190 this infectivity was modelled by the number of viraemic animals in the pen, for the qualitative

191 clinical-based dataset it was the number of animals in the pen presenting a CS $>0$, and for the 192 quantitative clinical-based dataset it was the sum of their CSs.

\subsection{Basic reproduction ratio estimation}

The R0 estimation method was adapted from Klinkenberg et al (2002). This method is based upon a SIR (Susceptible-Infected-Removed) model [2] of which $\alpha$ and $\beta$ parameters are estimated separately. The $\alpha$ parameter is the recovery rate of infectious animals, $1 / \alpha$ is thus the average duration of the infectious period and, more generally, $1 / \alpha$ represents the average total infectivity released by an infected animal. The $\beta$ parameter is the contact rate between animals, split here in two parameters: the within- and between-pen contact rates, denoted respectively $\beta_{\mathrm{w}}$ and $\beta_{\mathrm{b}}$. The within- and between-pen R0s are then, respectively: $\mathrm{R} 0_{\mathrm{w}}=\beta_{\mathrm{w}} / \alpha$, and $\mathrm{R} 0_{\mathrm{b}}=\beta_{\mathrm{b}} / \alpha$.

The $\beta$ s were estimated as described by Klinkenberg et al. (2002): a maximum

204 likelihood method was used, based upon a binomial distribution of the number $\mathrm{C}$ of animals 205 being contaminated in a given pen during a given time step:

$$
\mathrm{C} \sim \mathrm{B}\left[\mathrm{S}, 1-\exp \left(-\left(\beta_{\mathrm{w}} \mathrm{I}+\beta_{\mathrm{b}} \mathrm{J}\right) \quad / \mathrm{N}\right)\right] \text {, where: }
$$
animals in the pen at the beginning of the time step; 
$I$ and $J$ denote the total infectivity released during the time step by animals located in the same pen (I) and by animals located in the neighbouring pen (J); $\mathrm{B}[\mathrm{n}, \mathrm{p}]$ is the binomial distribution of parameters $\mathrm{n}$ and $\mathrm{p}$.

To estimate $\alpha$, Klinkenberg et al. (2002) used a general linear model for survival analysis with censoring. This approach is adopted when an animal is considered as either

214 infectious or not. The duration of the infectious period can then be modelled using survival 215 analysis techniques. However, this is not the case when infectivity is not a binary value such 216 as a viraemic status, but a numeric quantity such as a CS. Due to this and the fact that there was no censored data because animals were followed-up until the end of clinical signs and of viraemia, we used a simple non-parametric estimate of $1 / \alpha$ : the average total infectivity released by an individual over the course of the infection. For the viraemia-based dataset, the infectious period was the average duration of viraemia. The point estimate of $1 / \alpha$ (and thus of the R0s) is then computed as in Klinkenberg et al. (2002) assuming uncensored data. For the clinical-based dataset, it was either the average total CSs observed in infected animals (quantitative dataset) or the average number of days with a $\mathrm{CS}>0$ (qualitative dataset).

\subsection{Model comparison}

For the Eystrup strain, only the between-pen R0 was estimated (from experiment A data). For the Paderborn strain both R0s were estimated using data from experiments B (between-pen transmission) and $\mathrm{C}$ (within-pen transmission). Eystrup and Paderborn R0s estimates were computed separately from the virological- and clinical-based datasets.

231 known as Schwartz Information Criterion):

$$
\mathrm{BIC}=-2 \log (\mathrm{L})-(\mathrm{N}-\mathrm{k}-1) \log (\mathrm{N}), \text { where; }
$$

$233 \mathrm{~L}$ is the maximal likelihood obtained when estimating the $\beta \mathrm{s}$; 
$\mathrm{k}$ is the number of parameters of the model: $1\left(\beta_{\mathrm{b}}\right)$ for the Eystrup-specific models, and 2 $\left(\beta_{\mathrm{w}}\right.$ and $\beta_{\mathrm{b}}$ ) for the Paderborn-specific models;

$\mathrm{N}$ is the number of observations in the dataset (the number of non-infected animals) (Dohoo et al. 2003)

This criterion measures how closely the model fits the data (through the likelihood term L) and includes a penalty term that depends both on the number of parameters and on the size of the dataset. The smaller the value of the BIC, the better the model is. All the numerical analyses were conducted using R (R Development Core Team 2006).

\section{Results}

Most of the animals infected with the Eystrup strain (experiment A) died or had to be killed for welfare reasons, and a single (non-infected) animal survived (Table I). Except for this latter pig, the maximal observed CS was always $\geq 15$ (Figure 1). In experimentally inoculated animals, the average viraemia start day and the average clinical signs start day were identical (4 days PI). Viraemia and clinical signs started about 10 days later in contact infected animals (Table I).

No significant differences were observed between experimentally inoculated animals and contact infected pigs for the duration of viraemia, CS or for the average total CSs either in experiment A, nor in experiments B and C (Table I, Student's t-test: $\mathrm{p}>0.05$ ).

As expected, the Paderborn strain provoked a less marked mortality than the Eystrup strain (Table I). Maximal CSs were also lower: in experiment B, two animals showed a maximal $\mathrm{CS} \geq 15$ : an experimentally inoculated animal and a contact-infected pig. Both were 
slight and indefinite, such as a slightly reduced liveliness or a body shape indicating an empty stomach. However, at the end of the experiment, CSs were always zero and data censoring (that could bias R0 estimation) was considered negligible.

Total CSs were significantly higher with the Eystrup strain than with the Paderborn strain (Student's t-test: $\mathrm{p}=0.02$ ), but no significant effect of strain on CS duration was observed (Student't t-test: $\mathrm{p}>0.05$ ). Viraemia duration was significantly lower with the Eystrup strain than with the Paderborn strain (Student's t-test, $\mathrm{p}=0.01$ ).

The duration of viraemia was comparable in experiments B and C (Student's t-test: $\mathrm{p}>0.05$ ). Conversely, the duration of the clinical signs and the total CS were significantly higher in experiment $B$ than in experiment $C$ (Student's t-test, $p=0.04$ and $p=0.002$, respectively).

Following experimental inoculation with the Paderborn strain, average viraemia start date was 5 days PI in experiment B and 2 days PI in experiment C. In contact-infected animals, average viraemia start date was 19 days PI when contact was indirect (experiment B), and 13 days PI when contact was direct (experiment C) (Table I). The delay between average viraemia start date in experimentally inoculated animals and average viraemia start date in contact-infected pigs was thus 3 days longer with an indirect contact (experiment B: 14 days) than with a direct contact (experiment $\mathrm{C}: 11$ days).

Comparable results were observed for clinical signs. In experimentally inoculated animals, average clinical signs start date was 8 days PI in both experiments. In contactinfected animals, average clinical signs start date was 25 days PI when contact was indirect (experiment B), and 21 days PI when contact was direct (experiment C) (Table I). The delay between average clinical signs start date in experimentally inoculated animals and average clinical signs start date in contact-infected pigs was thus 4 days longer with an indirect contact (experiment B: 17 days) than with a direct contact (experiment C: 13 days). 
For the Eystrup strain, the estimated duration of the latent period was 4 days both for

285 the viraemia and for the clinical signs datasets. For the Paderborn strain, these estimates were 286 4 days for viraemia and 8 days for clinical signs. The duration of the latent periods were used to compute, for each contact-infected animal, an infection date. Figure 2 compares this reconstructed incidence of infections (number of animals contaminated per day) with the infectivity released, for each of the three experiments and for each of the three datasets. infected several days after all the experimentally infected animals had become viraemic. Contact animals were thus infected several days after the infectivity released by experimentally-inoculated animals had reached its maximal value. In experiments A and C, 294 all of the contact animals were infected before the earliest viraemia in the contact group, 295 demonstrating that all the contact animals were infected by the experimentally inoculated 296 pigs. In experiment B (indirect contact), a single contact animal (number 6909) was infected at D20, four days after the beginning of viraemia in two other contact infected pigs. This animal may thus have been infected either by experimentally inoculated pigs in the neighbouring pen, or by the contact infected pig in its own pen.

With the qualitative clinical-based dataset (Figure 2, middle row) when the contact 301 between experimentally inoculated and contact animals was indirect (experiments A and B), 302 contact animals were infected several days after the appearance of clinical signs in 303 experimentally inoculated animals. When the contact was direct (experiment C), the first 304 infections occurred earlier, as they coincided with the beginning of the clinical signs in 305 experimentally inoculated animals.

For the quantitative dataset based on clinical signs (Figure 2, bottom row), the 307 relationship between the peak in clinical signs in experimentally inoculated animals and 308 infection of contact animals appeared more variable. 
The average total infectivity $(1 / \alpha)$ estimated from the datasets based on viraemia and

310 that based on qualitative clinical signs were close, with higher values obtained for the

311 Paderborn strain (11-12 days) than for the Eystrup strain (8-9 days) (Table II). Conversely, for

312 the dataset based on quantitative clinical signs, the value obtained for the Eystrup strain was

313 twice as high as the value obtained for the Paderborn strain.

314 With the qualitative clinical signs-based dataset, Paderborn and Eystrup strains gave

315 similar between-pen contact rates (respectively 0.14 and 0.15 ). This was also the case for the

316 quantitative clinical signs-based dataset (respectively 0.04 and 0.02 ). Differences between the

317 strains were more marked for the viraemia-based dataset (Table II).

318 Whatever the dataset, between-pen R0 estimates obtained for the Eystrup strain were

319 close and ranged from 1.1 for the viraemia-based dataset, to 1.8 for the quantitative clinical-

320 based dataset. An intermediate value was obtained for the qualitative clinical-based dataset

321 (Table II). None of the Eystrup-specific $\mathrm{R} 0_{\mathrm{b}}$ were significantly $>1$. The value of the Bayes

322 Information Criterion (BIC) obtained for the quantitative clinical-based dataset was the

323 lowest, followed by the qualitative clinical-based dataset and finally by the viraemia-based

324 dataset (Table II).

325 The three datasets yielded similar values for the Paderborn-specific R $0_{\mathrm{b}}$ These

326 estimates were also close to those obtained for the Eystrup strain, and ranged from 1.0

327 (viraemia-based dataset) to 1.7 (quantitative clinical-based dataset). Again, these values were 328 not significantly $>1$.

329 Differences between datasets were observed for the Paderborn-specific within-pen R0:

330 estimated $\mathrm{R} 0_{\mathrm{w}}$ ranged from 4.0 for the viraemia-based dataset to 12.2 for the quantitative

331 clinical-based dataset. The value obtained from the qualitative clinical-based dataset was

332 again intermediate (Table II). Whatever the dataset, this Paderborn-specific $\mathrm{R} 0_{\mathrm{w}}$ was

333 significantly greater than 1 (lower bound of the $95 \%$ confidence interval $>1$ ). The lowest BIC 
was obtained for the qualitative clinical-based dataset, followed by the quantitative clinicalbased dataset and the viraemia-based dataset again had the highest BIC.

\section{Discussion}

Three CSFV transmission experiments conducted with two different strains (Eystrup and Paderborn) are reported here. The data obtained allowed us to study the effect of using different infectivity markers for transmission parameters estimation. Three different markers for individual infectivity were considered: viraemia data and clinical scores treated either qualitatively (presence/absence of clinical signs) or quantitatively. The three corresponding datasets allowed us to calculate R0 estimates for the Eystrup and for the Paderborn strains. The corresponding models were ranked using the value an information criterion (BIC), to evaluate the pertinence of the individual infectivity markers studied.

For experiments with both strains, the viraemia-based dataset always had the highest BIC value, and was thus the worst model. According to the BIC, the best model was, for the Eystrup strain, the quantitative clinical-based dataset and, for the Paderborn strain, the qualitative clinical-based dataset. However, for each strain, the BIC differences between this best model and the next one were not very large. According to Raftery (1996), evidence for superiority of the model with the lowest BIC is weak when absolute difference is $<2$, positive when absolute differences is $\geq 2$ and $<6$, and strong when the absolute difference is $\geq 6$. Following this interpretation, the BIC difference obtained for the Eystrup strain (6.4) would thus indicate strong evidence for superiority of the quantitative clinical-based dataset against the two others, which were similar. Similarly, the BIC difference obtained for the Paderborn strain (3) indicates the qualitative clinical-based dataset was superior to the two others, which were again similar to each other. The better fit of the clinical-based models suggests the existence of a relation between severity of the CSV symptoms and efficacy of CSFV 
transmission. In infected herds, the most severely affected animals could thus play a

360 prominent role in epizootic epidemic dynamic.

Furthermore, with the clinical-based datasets, estimated between-pen contact rates $\left(\beta_{\mathrm{b}}\right)$

362 appeared relatively independent of the strain as, for each dataset, the Eystrup-specific and the

363 Paderborn-specific estimates gave similar values. This was not the case for the viraemia-

364 based dataset. This suggests that, with the clinical-based datasets, the model allows a

365 separation between two kinds of disease transmission determinants: excretion determinants

366 (strain- and individual-specific) would be captured by the infectivity parameter $(1 / \alpha)$, whereas

367 infection determinants (contact structure between animals) would be captured by the contact

368 rates $(\beta)$. However, these results need to be confirmed by other transmission experiments with 369 different settings and different strains or animal types.

370 The R0 values obtained from the viraemia-based dataset using the Paderborn strain,

371 can be compared with estimates obtained earlier under comparable conditions (i.e. using 372 animals of comparable weights and a moderately virulent strain).

373 Laevens et al. (1999) conducted a transmission experiment in slaughter pigs, to study

374 the within-pen and the between-pen transmission of a moderately virulent CSFV strain 375 (isolate obtained from the 1st herd affected in the 1993-1994 Belgian epidemic). Using the 376 martingale estimator, Laevens et al. (1999) first reported a $\mathrm{R} 0_{\mathrm{w}}$ of 13.7 in slaughter pigs, with 377 a large confidence interval (standard error: 13.7, i.e. 95\% CI: -13.2-40). Klinkenberg et al. 378 (2002) later refined these estimates using a likelihood-based method to obtain a smaller 379 confidence interval for $\mathrm{R} 0_{\mathrm{w}}(15.5,95 \% \mathrm{CI}: 6.2-38.7)$ and to compute $\mathrm{R} 0_{\mathrm{b}}: 3.4$ (95\% CI: $1.5-$ 380 7.4). All of these estimates were obtained using measurements of viraemia.

381 Data reported here show less marked differences between the efficacy of indirect 382 contact (experiment B) and the efficacy of direct contact (experiment C) for CSFV 383 transmission. The delay between average viraemia start in experimentally infected animals 
and in contact infected pigs was 11 days with direct contact (experiment $\mathrm{C}$ ) and 14 days with indirect contact. Despite this relatively small difference ( 3 days), $\mathrm{R} 0_{\mathrm{w}}$ estimate is significantly $>1$ whereas $R 0_{b}$ is not. This result is explained by the fact that, in experiment $\mathrm{B}$ (indirect contact), one of the four contact pigs was infected after some of the animals located in the same pen had started their viraemia: this latter pig may thus have been infected through within-pen contagion. Nevertheless, the $\mathrm{R} 0_{\mathrm{w}}$ and $\mathrm{R} 0_{\mathrm{b}}$ we obtained from the viraemia-based 390 dataset, Paderborn strain (respectively 4.0 and 1.0) were clearly lower than the previously published estimates. Confidence intervals are large and this difference may be attributed to stochastic variations. Differences between the strains may also be incriminated. Moreover, concerning $\mathrm{R} 0_{\mathrm{b}}$, the Laevens et al. (1999) estimate uncovered two transmission modalities

394 (airborne transmission and transmission by contaminated clothes), whereas in the present 395 case, only airborne transmission was possible. This design difference could also partially 396 explain the $\mathrm{R} 0_{\mathrm{b}}$ estimates variations. Finally, it is interesting to note that the $\mathrm{R} 0_{\mathrm{w}}$ values obtained from the clinical-based datasets (7.6 and 12.2 for the qualitative and quantitative datasets) are closer to prior estimates than the values obtained from the viraemia-based dataset.

In recent years, detailed research studies have been conducted to design and enhance 401 R0 estimation methods while adapting them to the type of data produced by transmission experiments: accurate (as many biological indicators can be measured) and sparse (since, for 403 large animals such as pigs, the number of studied individuals is always low). Such studies 404 allowed us to calculate more accurate R0s, with smaller confidence intervals. However, 405 besides this methodological research, little attention has been paid to the nature of the data 406 used to estimate R0. Basic reproduction ratio aims to quantify virus transmission. In principle, its 408 estimation could thus be based upon any direct or indirect infectivity marker. Previous studies 
409 have used viraemia-based markers, and Dewulf et al. (2002) compared viraemia and PCR

410 based R0s in vaccinated sows, obtaining markedly different estimates. We chose to compare

411 the usual viraemia-based estimates with two clinical-based estimates, using a CSF-specific

412 score system for symptoms severity. The basic hypothesis is that the link between the (non-

413 measurable) infectivity and the clinical scores is as close as the link between infectivity and

414 viraemia. A better goodness of fit suggests that the clinical-based approach represents a cost-

415 effective alternative to the viraemia-based approach.

416

417

418

419 Acknowledgements

420 This work was partly granted by a PhD thesis grant from the "Conseil Regional de Bretagne".

421 The authors thank Valérie Rose and Stéphane Gorin for their excellent technical assistance,

422 Bruno Jan for animal handling, A. Mahé for samples collection, and Linda Dixon and Emma

423 Fishbourne for rewording the document.

424 


\section{References}

426 Alexandersen S., Quan M., Murphy C., Knight J. and Zhang Z., 2003. Studies of quantitative parameters of virus excretion and transmission in pigs and cattle experimentally infected with foot-and-mouth disease virus. J. Comp. Pathol. 129, 268-282.

Anderson R.M. and May R.M., 1991. Infectious diseases of humans: dynamics and control, Oxford university press, United Kingdom.

Anonym, 2002. Commission Decision of February 2002 approving a diagnostic manual establishing diagnostic procedures, sampling methods and criteria for evaluation of the laboratory tests for the confirmation of Classical Swine Fever (2002/106/EC), Chapter VII. Off. J. Eur. Union L039, 71-88.

Anonym, 2007. Animal disease notification system. Annual report 2006. European commission, $63 \mathrm{pp}$.

Becker N.G., 1989. Analysis of infectious disease data. Monographs on Statistics and Applied Probability, Chapman and Hall, London.

Dahle J. and Liess B., 1992. A review on classical swine fever infections in pigs: epizootiology, clinical disease and pathology. Comp. Immunol. Microbiol. Infect. Dis. $15,203-211$.

De Jong M.C.M. and Kimman T.G., 1994. Experimental quantification of vaccine-induced reduction in virus transmission. Vaccine $12,761-766$.

444 Depner K.R., Hinrichs U., Bickhardt K., Greiser-Wilke I., Pohlenz J., Moennig V. and Liess B., 1997. Influence of breed-related factors on the course of classical swine fever virus infection. Vet. Rec. 140, 506-507.

Dewulf J., Laevens H., Koenen F., Mintiens K. and De Kruif A., 2001. An experimental infection with classical swine fever virus in pregnant sows: transmission of the virus, 
course of the disease, antibody response and effects on gestation. J. Vet. Med. B 48, 593-591.

Dewulf J., Laevens H., Koenen F., Mintiens K. and De Kruif A., 2002. An E2 subunit marker vaccine does not prevent horizontal or vertical transmission of classical swine fever virus. Vaccine 20, 86-96.

Dohoo I., Martin W. and Stryhn H., 2003. Veterinary Epidemiologic research. AVC Inc., Chalottetown, Canada.

Elbers A.R., Vos J.H., Bouma A. and Stegeman J.A., 2004. Ability of veterinary pathologists to diagnose classical swine fever from clinical signs and gross pathological findings. Prev. Vet. Med. 66, 239-246.

Elbers A.R.W., Stegeman A., Moser H., Ekker H.M., Smak J.A. and Pluimers F.H., 1999. The classical swine fever epidemic 1997-1998 in the Netherlands: Descriptive epidemiology. Prev. Vet. Med. 42, 157-184.

Engel B., Bouma A., Stegeman A., Buist W., Elbers A., Kogut J., Dopfer D. and de Jong M.C., 2005. When can a veterinarian be expected to detect classical swine fever virus among breeding sows in a herd during an outbreak? Prev. Vet. Med. 67, 195-212.

Floegel-Niesmann G., Bunzenthal C., Fischer S. and Moennig V., 2003. Virulence of recent and former classical swine fever virus isolates evaluated by their clinical and pathological signs. J. Vet. Med. B. 50, 214-220.

Fritzemeier J., Teuffert J., Greiser-Wilke I, Staubach C., Schluter H. and Moennig V., 2000. Epidemiology of classical swine fever in Germany in the 1990s. Vet. Microbiol. 77, $29-41$.

Fröhlich K., Thiede S., Kozikowski T. and Jakob W., 2002. A review of mutual transmission of important infectious diseases between livestock and wildlife in Europe. Ann. N. Y. Acad. Sci. 969, 4-13.Klinkenberg D., De Bree J., Laevens H. and De Jong M.C.M., 
2002. Within- and between-pen transmission of Classical Swine Fever Virus: a new

475

476

477

478

479

480

481

482

483

484

485

486

487

488

489

490

491

492

493

494

495

496

497

498 method to estimate the basic reproduction ratio from transmission experiments. Epidemiol. Infect. 128, 293-299

Koenen F., Van Caenegem G., Vermeersch J.P., Vandenheede J. and Deluyker H. 1996. Epidemiological characteristics of an outbreak of classical swine fever in an area of high pig density. Vet. Rec. 139, 367-371.

Kroese A.H. and De Jong M.C.M., 2001. Design and analysis of transmission experiments. In: Society For Veterinary and Preventive Medicine, Noordwijekerhout, The Netherlands, 21-37.

Laevens, H., Koenen, F., Deluyker, H., Berkvens, D., de Kruif, A., 1998. An experimental infection with classical swine fever virus in weaner pigs. I. Transmission of the virus, course of the disease, and antibody response. Vet. Q. 20, 41-45.

Laevens, H., Koenen, F., Deluyker, H., Dekruif, A., 1999. Experimental infection of slaughter pigs with classical swine fever virus: transmission of the virus, course of the disease and antibody response. Vet. Rec. 145, 243-248.

Meuwissen M.P., Horst S.H., Huirne R.B. and Dijkhuizen A.A., 1999. A model to estimate the financial consequences of classical swine fever outbreaks: principles and outcomes. Prev. Vet. Med. 42, 249-270.

Mittelholzer C., Moser C., Tratschin J.D. and Hofmann A., 2000. Analysis of classical swine fever virus replication kinetics allows differentiation of highly virulent from avirulent strains. Vet. Microbiol. 74, 293-3.8.

Moennig, V., 2000. Introduction to classical swine fever: virus, disease and control policy. Vet. Microbiol. 73, 93-102.

Moennig V., Floegel-Niesmann G. and Greiser-Wilke I., 2003. Clinical signs and epidemiology of classical swine fever: a review of new knowledge. Vet. J. 165, 11-20. 
Quan M., Murphy C.M., Zhang Z. and Alexandersen S., 2004. Determinants of early footand-mouth disease virus dynamics in pigs. J. Comp. Pathol. 131, 294-307.

501 R Development Core Team, 2006. R: A Language and Environment for Statistical Computing. R Foundation for Statistical Computing. Vienna, Austria, 2006, URL: http://www.R-project.org.

Raftery A.E., 1996. Bayesian model selection in social research. In: Marsden P.V. (ed.) Sociological methodology. Blackwell, Oxford, United Kingdom, 111-163.

Saatkamp H.W., Berentsen P.B. and Horst H.S., 2000. Economic aspects of the control of classical swine fever outbreaks in the European Union. Vet. Microbiol. 73, 221-237.

508 Sandvik T., Drew T. and Paton D., 2000. CSF virus in East Anglia: where from? Vet. Rec. $147,251$.

510 Terpstra C. and De Smit A.J., 2000. The 1997/1998 epizootic of swine fever in the Netherlands: Control strategies under a non-vaccination regimen. Vet. Microbiol. 77, 3-15.

513 Wood L., Brockman S., Harkness J.W. and Edwards S., 1998. Classical swine fever: virulence and tissue distribution of a 1986 English isolate in pigs. Vet. Rec. 122, 391394. 
516 Table I. Estimated CSFV viraemic periods and observed CSF clinical scores in three

517 transmission experiments conducted with a highly (A) or a moderately (B and C) virulent

518 strain, and with direct $(\mathrm{C})$ or indirect (A and B) contacts between experimentally inoculated

519 and contact animals.

\begin{tabular}{|c|c|c|c|c|c|c|c|c|c|c|c|}
\hline \multirow[t]{2}{*}{ Exp. } & \multirow[t]{2}{*}{ Strain } & \multirow[t]{2}{*}{ Pen } & \multirow[t]{2}{*}{ Animal } & \multirow[t]{2}{*}{ Death $^{a}$} & \multicolumn{3}{|c|}{ Viraemic period $^{b}$} & \multicolumn{4}{|c|}{ Clinical scores $^{\mathrm{c}}$} \\
\hline & & & & & Start & End & Duration & Start & End & Duration & Total \\
\hline \multirow[t]{11}{*}{$\mathrm{A}$} & \multirow[t]{6}{*}{ Eystrup } & 1 & 6885 & 11 & 5 & 11 & 7 & 4 & 11 & 8 & 79 \\
\hline & & 1 & 6889 & 10 & 5 & 10 & 6 & 4 & 10 & t & 60 \\
\hline & & 1 & 6900 & 12 & 2 & 12 & 11 & 4 & 12 & 9 & 97 \\
\hline & & 1 & 6899 & 10 & 5 & 10 & 6 & 3 & 10 & 8 & 58 \\
\hline & & \multirow[t]{2}{*}{1} & 6913 & 10 & 2 & 10 & 9 & 4 & 10 & 7 & 54 \\
\hline & & & Average & & 4 & 11 & 8 & 4 & 11 & 8 & 70 \\
\hline & \multirow[t]{5}{*}{ Contact } & 2 & 6895 & \multirow[t]{2}{*}{21} & 14 & 21 & 8 & 14 & 21 & 8 & 101 \\
\hline & & 2 & 6903 & & 14 & 23 & 10 & 14 & 27 & 14 & 94 \\
\hline & & 2 & 6915 & 27 & 16 & 27 & 12 & 14 & 27 & 14 & 189 \\
\hline & & \multirow[t]{2}{*}{2} & 6916 & 21 & 16 & 21 & 6 & 14 & 21 & 8 & 85 \\
\hline & & & Average & & 15 & 23 & 9 & 14 & 24 & 11 & 117 \\
\hline \multirow[t]{11}{*}{ B } & \multirow[t]{6}{*}{ Paderborn } & 1 & 6884 & \multirow[t]{2}{*}{30} & 5 & 23 & 19 & 6 & 30 & 16 & 140 \\
\hline & & 1 & 6887 & & 5 & 15 & 11 & 9 & 34 & 20 & 65 \\
\hline & & 1 & 6898 & \multirow{3}{*}{$\begin{array}{l}21 \\
14\end{array}$} & 5 & 21 & 17 & 14 & 21 & 8 & 77 \\
\hline & & 1 & 6902 & & 5 & 14 & 10 & 6 & 14 & 9 & 45 \\
\hline & & \multirow[t]{2}{*}{1} & 6907 & & 5 & 15 & 11 & 5 & 38 & 34 & 119 \\
\hline & & & Average & & 5 & 18 & 14 & 8 & 27 & 17 & 89 \\
\hline & \multirow[t]{5}{*}{ Contact } & 2 & 6894 & & 16 & 29 & 14 & 25 & 36 & 12 & 50 \\
\hline & & 2 & 6886 & 37 & 16 & 32 & 17 & 25 & 37 & 13 & 148 \\
\hline & & 2 & 6906 & & 19 & 29 & 11 & 26 & 36 & 11 & 39 \\
\hline & & 2 & 6909 & & 24 & 29 & 6 & 26 & 36 & 11 & 38 \\
\hline & & & Average & ) & 19 & 30 & 12 & 25 & 36 & 12 & 69 \\
\hline \multirow[t]{12}{*}{$\mathrm{C}$} & \multirow[t]{5}{*}{ Paderborn } & 1 & 7187 & 15 & 2 & 15 & 14 & 7 & 15 & 9 & 35 \\
\hline & & 1 & 7227 & 18 & 2 & 18 & 17 & 10 & 18 & 9 & 31 \\
\hline & & 2 & 7214 & & 2 & 7 & 6 & 8 & 24 & 3 & 3 \\
\hline & & 2 & 7239 & & 2 & 12 & 11 & 8 & 42 & 13 & 24 \\
\hline & & & Average & & 2 & 13 & 12 & 8 & 25 & 8 & 23 \\
\hline & \multirow[t]{7}{*}{ Contact } & 1 & 7199 & \multirow{6}{*}{25} & 13 & 27 & 15 & 18 & 33 & 14 & 16 \\
\hline & & 1 & 7216 & & 16 & 16 & 1 & 24 & 30 & 2 & 2 \\
\hline & & 1 & 7241 & & 13 & 25 & 13 & 15 & 25 & 10 & 33 \\
\hline & & 2 & 7189 & & 13 & 21 & 9 & 23 & 42 & 11 & 17 \\
\hline & & 2 & 7203 & & 13 & 21 & 9 & 15 & 33 & 5 & 5 \\
\hline & & 2 & 7234 & & 13 & 27 & 15 & 30 & 30 & 1 & 1 \\
\hline & & & Average & & 13 & 23 & 10 & 21 & 32 & 7 & 12 \\
\hline
\end{tabular}

$520 \quad{ }^{a}$ Animals killed for welfare reasons.

${ }^{\mathrm{b}}$ Linearly interpolated from results of CSFV isolation from blood samples taken at days $0,3,5,7,10,12,14,17,19,21,24$, 26, 28 (experiment A); 0, 3, 5, 7, 10, 12, 14, 17, 19, 21, 24, 26, 28, 31, 33, 35, 38, 40, 42 (experiment B); and 0, 3, 7, 9, 11, $14,16,18,12,23,25,27,28,31,35,38,45$ (experiment C).

${ }^{\mathrm{c}}$ Measured daily. Start, end: first and last day for which clinical score is $>0$. Duration: number of days for which clinical score is $>0$. Total: global sum of clinical scores. 
526 Table II. Comparison of the basic reproduction ratios estimated from results of CSFV

527 isolation from blood (viraemia-based dataset) or from clinical scores (qualitative and 528 quantitative clinical-based datasets) observed in three transmission experiments conducted 529 with a highly (Eystrup) or a moderately (Paderborn) virulent strain and with direct or indirect 530 contacts between experimentally inoculated and contact animals.

\begin{tabular}{llcccc}
\hline Strain & Parameter & & Viraemia-based & \multicolumn{2}{c}{ Clinical-based dataset } \\
\cline { 5 - 6 } & & & dataset & Qualitative & Quantitative \\
\hline Eystrup & Latent period & & 4 & 4 & 4 \\
& Avg. total infectivity & $1 / \alpha$ & $8.3(6.6-10.1)$ & $9.2(7.1-11.4)$ & $90.8(59.4-122.2)$ \\
& Between-pen contact rate & $\beta_{\mathrm{b}}$ & $0.13(0.05-0.35)$ & $0.15(0.06-0.40)$ & $0.02(0.01-0.05)$ \\
& Between-pen R0 & $\mathrm{R} 0_{\mathrm{b}}$ & $1.1(0.4-2.9)$ & $1.4(0.5-3.7)$ & $1.8(0.7-4.8)$ \\
& Bayes Information Criterion & $\mathrm{BIC}$ & 21.6 & 20.4 & 14.0 \\
\hline Paderborn & Latent period & & 4 & 8 & 8 \\
& Avg. total infectivity & $1 / \alpha$ & $11.9(9.7-14.1)$ & $11.1(7.6-14.6)$ & $46.7(25.1-68.4)$ \\
& Within-pen contact rate & $\beta_{\mathrm{b}}$ & $0.33(0.16-0.71)$ & $0.69(0.31-1.53)$ & $0.26(0.12-0.58)$ \\
& Between-pen contact rate & $\beta_{\mathrm{w}}$ & $0.08(0.03-0.25)$ & $0.14(0.05-0.39)$ & $0.04(0.01-0.10)$ \\
& Within-pen R0 & $\mathrm{R} 0_{\mathrm{w}}$ & $4.0(1.9-8.4)$ & $7.6(3.4-17.0)$ & $12.2(5.5-27.3)$ \\
& Between-pen R0 & $\mathrm{R} 0_{\mathrm{b}}$ & $1.0(0.3-3.0)$ & $1.6(0.6-4.3)$ & $1.7(0.6-4.6)$ \\
Bayes Information Criterion & $\mathrm{BIC}$ & 49.1 & 44.6 & 47.6 \\
\hline
\end{tabular}

Bracketed: $95 \%$ confidence intervals. 
533 Figure 1. Individual clinical scores (lines) and results of CSFV isolation from blood (filled

534 circle: positive, empty circle: negative) in three transmission experiments conducted with a

535 highly (A) or a moderately (B and C) virulent strain, and with direct (C) or indirect (A and B)

536 contacts between experimentally inoculated and contact animals.

Clinical scores: measured daily. CSFV isolation from blood: samples taken at days $0,3,5,7,10,12,14,17,19,21,24,26$, 28 (experiment A); 0, 3, 5, 7, 10, 12, 14, 17, 19, 21, 24, 26, 28, 31, 33, 35, 38, 40, 42 (experiment B); and 0, 3, 7, 9, 11, 14, $16,18,12,23,25,27,28,31,35,38,45$ (experiment $\mathrm{C}$ ). Stars: animals killed for welfare reasons.

543 Figure 2. Reconstruction of the animals' infectivity (left axis, solid line: experimentally

544 inoculated, dashed: contact-infected) and of the contact animals' infection (bars, right axis)

545 based upon results of CSFV isolation from blood (viraemia-based dataset, top) or upon

546 clinical scores (clinical-based dataset, middle: qualitative, bottom: quantitative) observed in

547 three transmission experiments conducted with a highly (A) or a moderately (B and C)

548 virulent strain, and with direct (C) or indirect (A and B) contacts between experimentally

549 inoculated and contact animals.

550 Infection of contact animals is assumed to occur 4 days before the $1^{\text {st }}$ CSFV isolation from blood (top) or the $1^{\text {st }}$

551 non-zero clinical score (middle, bottom, A), and 8 days before the $1^{\text {st }}$ non-zero clinical score (middle, bottom, B,

552 C) 

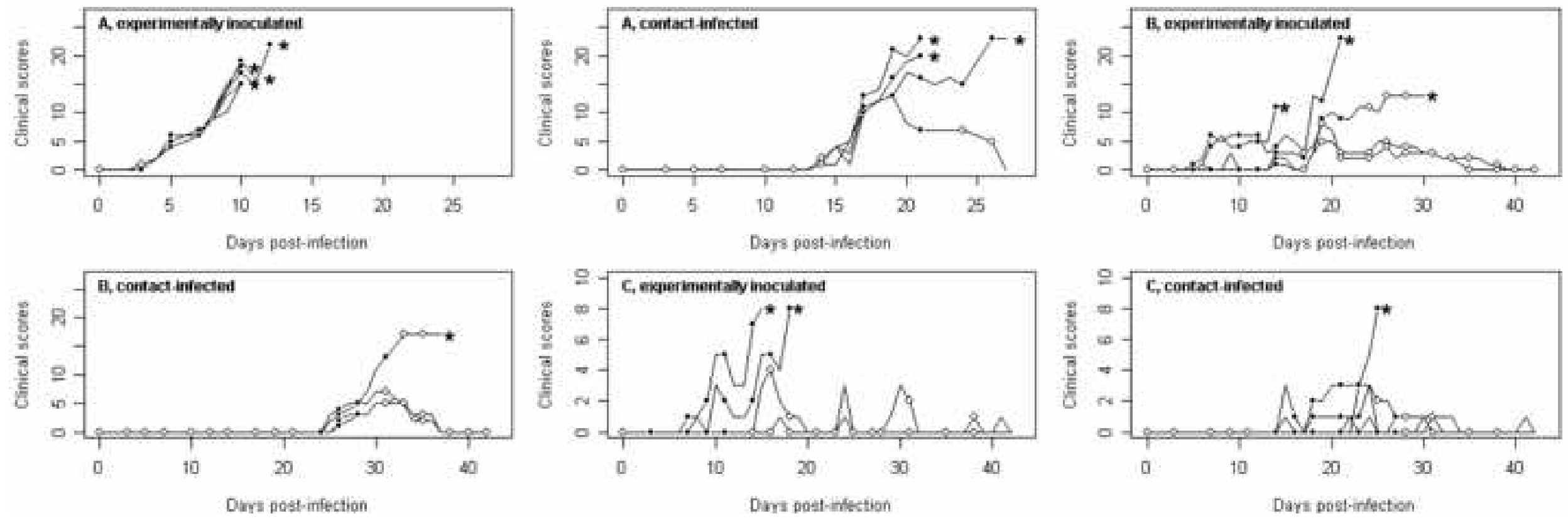

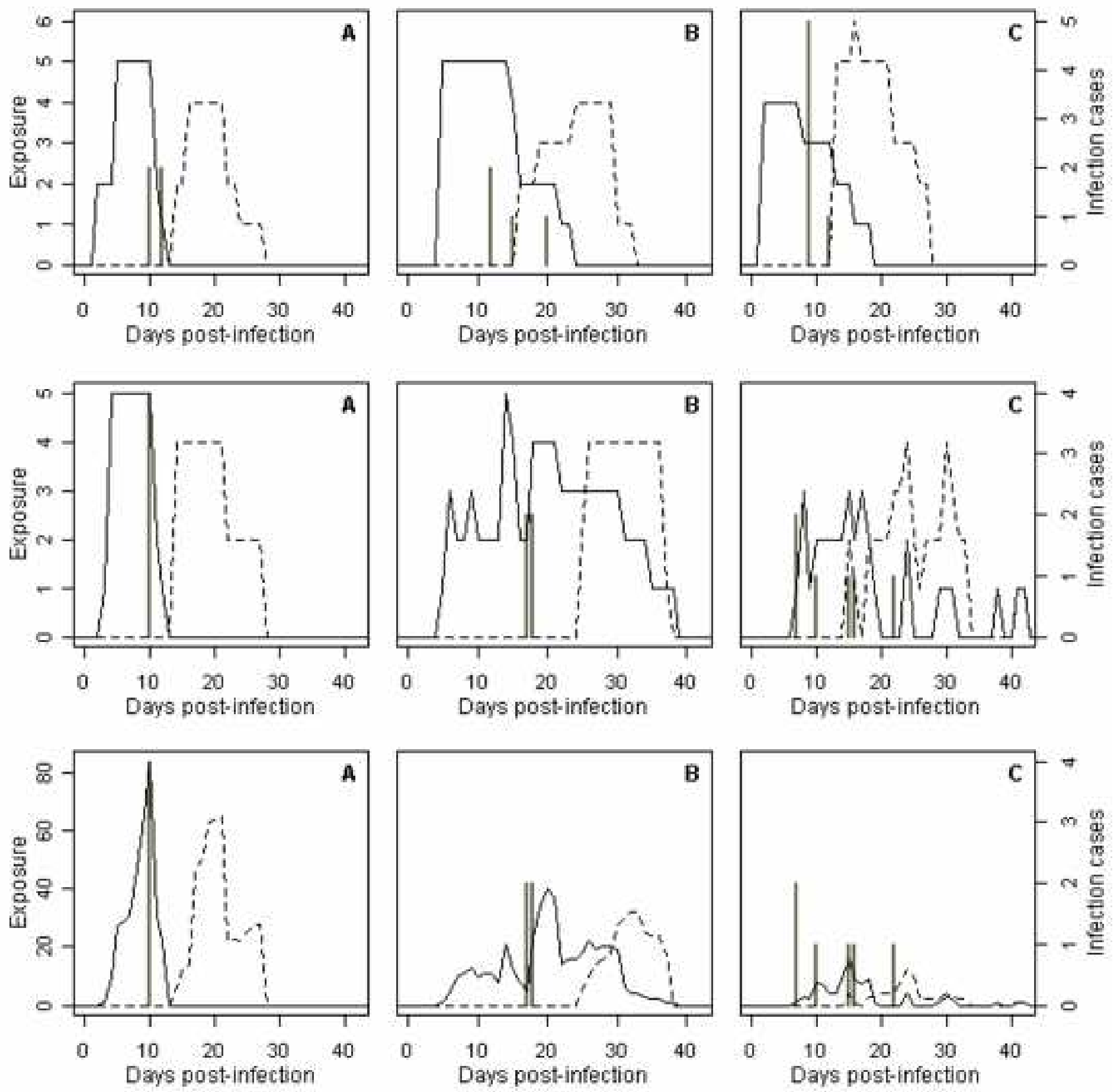

rage $2 /$ or $2 /$ 